\title{
A novel human mAb (MERS-GD27) provides prophylactic and postexposure efficacy in MERS-CoV susceptible mice
}

\author{
Peihua $\mathrm{Niu}^{1 \dagger}$, Guangyu Zhao ${ }^{2 \dagger}$, Yao Deng ${ }^{1}$, Shihui $\mathrm{Sun}^{2}$, Wenling Wang ${ }^{1}$, Yusen $\mathrm{Zhou}^{2 *} \&$ \\ Wenjie $\operatorname{Tan}^{1 *}$ \\ ${ }^{1}$ National Institute for Viral Disease Control and Prevention, Chinese Center for Disease Control and Prevention, Beijing 102206, China; \\ ${ }^{2}$ State Key Laboratory of Pathogen and Biosecurity, Beijing Institute of Microbiology and Epidemiology, Beijing 100850, China
}

Received May 21, 2018; accepted June 16, 2018; published online August 7, 2018

Citation: Niu, P., Zhao, G., Deng, Y., Sun, S., Wang, W., Zhou, Y., and Tan, W. (2018). A novel human mAb (MERS-GD27) provides prophylactic and postexposure efficacy in MERS-CoV susceptible mice. Sci China Life Sci 61, 1280-1282. https://doi.org/10.1007/s11427-018-9343-8

Dear Editor,

Since September 2012, the Middle East respiratory syndrome coronavirus (MERS-CoV) cases have been reported in more than 27 countries, and more than 2,000 cases have been confirmed in the laboratory (http://www.who.int/ emergencies /mers-cov/en/). MERS-CoV causes an acute and severe respiratory illness with a high mortality rate $(\sim 35 \%)$ in humans (Shi et al., 2017, Zaki et al., 2012). Neutralizing antibodies targeting the spike of MERS-CoV have been shown to be a therapeutic option for treatment of lethal disease (Agrawal et al., 2016, Ying et al., 2014).

In our study, we characterized a novel human monoclonal antibody (named MERS-GD27) in our human DPP4 (hDPP4) transgenic mouse model of MERS-CoV and the evaluation results showed that MERS-GD27 had preventive and therapeutic protective effects in mice before and after a lethal challenge with MERS-CoV.

The MERS-GD27 was generated from a recovered natural MERS-CoV infection patient according to the method (Smith et al., 2009). The heavy and light chain DNA from each B cell was cloned into separate vectors. A double gene expression vector transiently transfecting into human embryonic kidney (HEK)-293FS cells was used to produce the

$\dagger$ Contributed equally to this work

*Corresponding authors (Yusen Zhou, email: yszhou@bmi.ac.cn; Wenjie Tan, email: tanwj28@163.com) antibody. The jetPRIME transfection reagent (Polyplustransfection $^{\circledR}$, France) was used with equimolar amounts of heavy and light chain vector according to the standard protocol. The antibody was purified using a protein $\mathrm{G}$ column (GE Healthcare, USA) and protein purity was estimated by SDS-polyacrylamide gel electrophoresis and protein concentration was measured spectrophotometrically (Nanodrop Technologies Inc., USA). MERS-GD27 targeted the DPP4binding site on the receptor-binding domain (RBD) with an $\mathrm{IC}_{50}$ value of $0.0010 \mu \mathrm{g} \mathrm{mL}^{-1}$ in pseudovirus neutralization experiments, and showed subnanomolar affinity for the MERS-CoV S protein (equilibrium dissociation constant $\left(K_{\mathrm{D}}\right)$ equivalent to $\left.0.775 \mathrm{nmol} \mathrm{L}^{-1}\right)$. When neutralizing activity was tested by Plaque Reduction Neutralization Test (PRNT) using the live MERS-CoV stock (hCoV-EMC), MERS-GD27 demonstrated potent inhibitory activity against MERS-CoV infection with concentrations of $0.001 \mu \mathrm{g} \mathrm{well}^{-1}$, and the inhibition rates were $60 \%$ (Figure S1 in Supporting Information).

Using the hDPP4 transgenic mice as MERS-CoV susceptible animal model (Qiu et al., 2016, Zhao et al., 2013), we investigated the prophylactic protective efficacy and therapeutic potential of MERS-GD27 in vivo. To accomplish this, each group $(n=8)$ of mice was treated via the tail intravenous injection route with $5 \mathrm{mg} \mathrm{kg}^{-1}$ MERS-GD27 diluted in $300 \mu \mathrm{L}$ PBS, and intranasally challenged at $24 \mathrm{~h}$ post- and pre-treatment with three $\mathrm{LD}_{50}$ of MERS-CoV in a 
volume of $20 \mu \mathrm{L}$. The control group was treated with a high dose $\left(50 \mathrm{mg} \mathrm{kg}^{-1}\right)$ of irrelevant monoclonal antibody (antiHA of influenza virus). Challenged mice were monitored daily for clinical manifestations (weight loss) and survival. As shown in Figure 1A, the prevention group survived at $60 \%$ showing no distinct clinical symptoms after viral infection. The therapy group survived at $40 \%$ initially showed a gradual weight loss $(0 \%-20 \%)$ until day 11 just before starting to quickly recover (Figure 1Ai). All surviving mice continued to recover and appeared well up to $14 \mathrm{dpi}$ when the experiment was terminated (Figure 1Aii). All MERS-CoVchallenged mice treated with irrelevant monoclonal antibody exhibited profound weight loss $(>20 \%)$ and succumbed to infection with $100 \%$ mortality by day 11 p.i. (Figure $1 \mathrm{Ai}$, ii).

We also investigated the lung virus titers and RNA copy in challenged mice at day 5 after virus challenge. Specifically, the lung specimens were harvested for determining viral titers of MERS-CoV by Vero E6 cell-based infectivity assay and Real time quantitative PCR assay (qRT-PCR). As shown in Figure $1 \mathrm{Bi}$, the lung viral titers among mice treated with MERS-GD27 pre- or post-viral challenge were significantly lower $(P<0.001)$ compared to control group. In addition, viral RNA loads among mice treated with MERS-GD27 preor post-viral challenge were also significantly lower than those in the control group (Figure 1Bii). These data indicated that MERS-GD27 conferred significant protection for mice

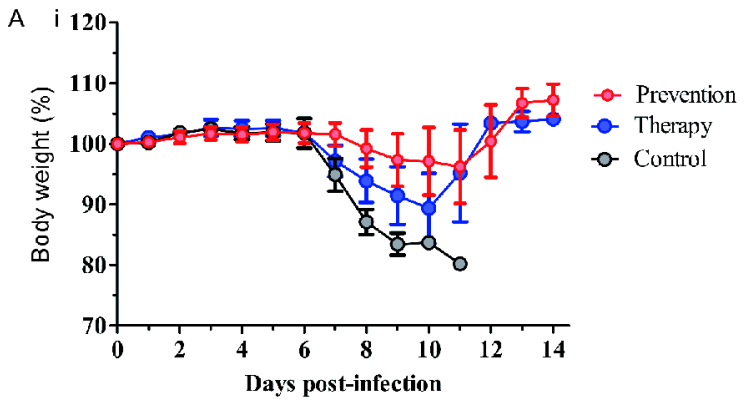

$\mathrm{B}$

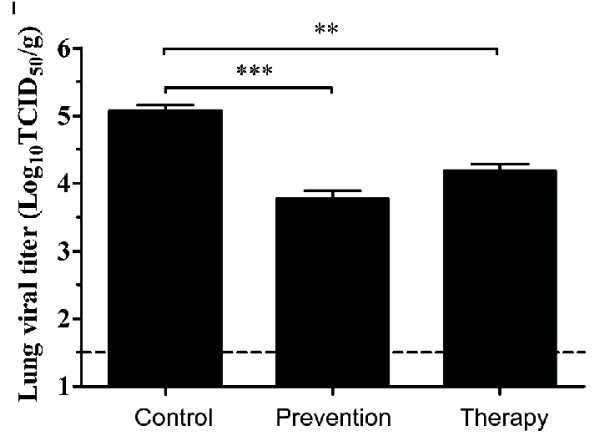

when administered pre- or post-viral challenge.

MERS-GD27 could effectively protect in both prophylactic and postexposure settings in vivo. Thus, MERS-GD27 was highly promising as a potent inhibitor for urgent prophylaxis in adjunctive treatment for patients infected with MERS-CoV. We noted that passively transferred with $5 \mathrm{mg} \mathrm{kg}^{-1}$ of MERS-GD27 to mice $24 \mathrm{~h}$ prior to challenge with three $\mathrm{LD}_{50}$ of MERS-CoV resulted in $60 \%$ protection against lethality. Although using $5 \mathrm{mg} \mathrm{kg}^{-1}$ MERS-GD27 as a prophylaxis was suboptimal to completely neutralize viral infection, the protective effect was obviously presented compared to control groups, especially to the recovery of bodyweight loss and the reduction of viral loads, as shown in Figure 1. The therapeutic efficacy of MERS-GD27 was similar to the prophylactic studies, administration of MERSGD27 at a concentration of $5 \mathrm{mg} \mathrm{kg}^{-1}$ at $24 \mathrm{~h}$ after MERS$\mathrm{CoV}$ challenge provided $40 \%$ protection, against infectioninduced lethality, accompanied by reduced viral loads (both infectious virus and viral RNA) within the lungs. These data demonstrated that MERS-GD27 was an effective therapeutic agent for MERS-CoV infection.

Taken together, these results indicated that the MERS-CoV specific human monoclonal antibody could be highly effective as prophylactic or therapeutic modalities in protecting transgenic mice against MERS-CoV infection. In the future, more exploration and research need to be performed on
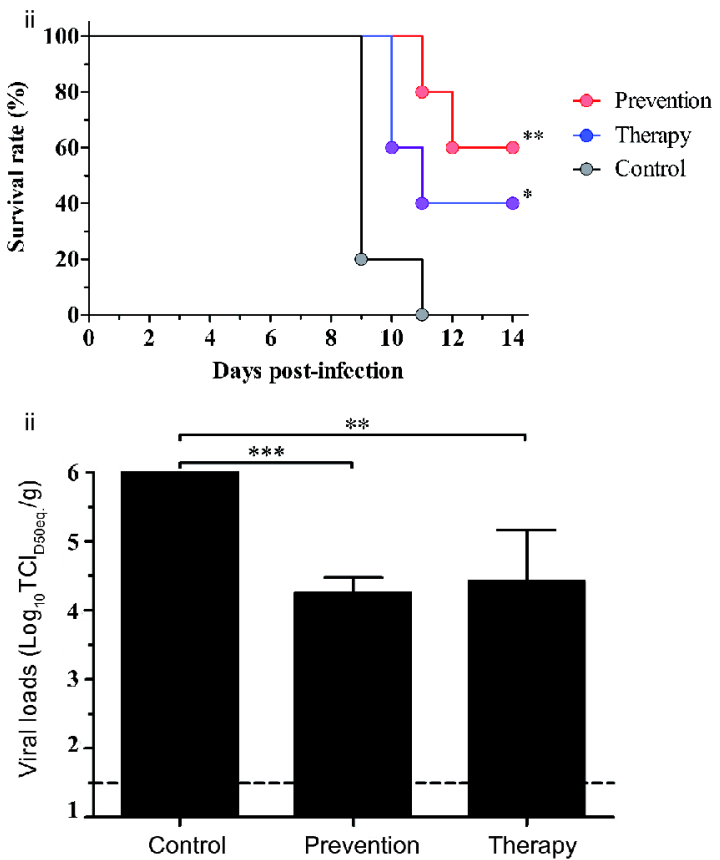

Figure 1 The functional verification of MERS-GD27 in MERS-CoV susceptible mice. A, Prophylactic and therapeutic efficacy of MERS-GD27 in protecting susceptible mice against lethal dose MERS-CoV challenge. Challenged mice were monitored daily for the weight loss (i) and accumulated mortality (ii), expressed as percent (\%) body weight and survival rate, respectively. B, Treatment with MERS-GD27 significantly inhibited MERS-CoV infection within the lungs. Lung specimens collected at day 5 after viral challenge were processed for assessing the viral titers by using both Vero E6-based infectivity assay and qRT-PCR targeting N3 gene of MERS-CoV, and expressed as $\log _{10} \mathrm{TCID}_{50} / \mathrm{g}$ and $\log _{10} \mathrm{TCID}$ (0) equivalent (eq.)/g, respectively. (i) Prophylactic and therapeutic efficacy of MERS-GD27 treatment in reducing the lung titers of infectious virus. (ii) Prophylactic and therapeutic efficacy of MERS-GD27 in reducing the titers of viral RNA. The data were representative of at least two independently conducted assays using the same samples. Data were presented as mean \pm standard error (SE). *,** and $* * *, P<0.001$ as determined by using Student's $t$ test. 
therapeutic doses, inoculation times, vaccination methods, and so on.

Compliance and ethics The author(s) declare that they have no conflict of interest.

Acknowledgements We thank Dr. Bart L. Haagmans and Dr. Ron A.M. Fouchier (Erasmus MC, Rotterdam, the Netherlands) for providing MERS$\mathrm{CoV}$ (isolate hCoV-EMC/2012). This work was supported by the National Key Research and Development Program of China (2016YFD0500300 to Wenjie Tan). The funders had no role in study design, data collection and analysis, decision to publish, or preparation of the manuscript.

Agrawal, A.S., Ying, T., Tao, X., Garron, T., Algaissi, A., Wang, Y., Wang, L., Peng, B.H., Jiang, S., Dimitrov, D.S., et al. (2016). Passive transfer of A germline-like neutralizing human monoclonal antibody protects transgenic mice against lethal middle east respiratory syndrome coronavirus infection. Sci Rep 6, 31629.

Qiu, H., Sun, S., Xiao, H., Feng, J., Guo, Y., Tai, W., Wang, Y., Du, L., Zhao, G., and Zhou, Y. (2016). Single-dose treatment with a humanized neutralizing antibody affords full protection of a human transgenic mouse model from lethal Middle East respiratory syndrome (MERS)coronavirus infection. Antiviral Res 132, 141-148.

Shi, W., Li, J., Zhou, H., and Gao, G.F. (2017). Pathogen genomic surveillance elucidates the origins, transmission and evolution of emerging viral agents in China. Sci China Life Sci 60, 1317-1330.

Smith, K., Garman, L., Wrammert, J., Zheng, N.Y., Capra, J.D., Ahmed, R., and Wilson, P.C. (2009). Rapid generation of fully human monoclonal antibodies specific to a vaccinating antigen. Nat Protoc 4, 372-384.

Ying, T., Du, L., Ju, T.W., Prabakaran, P., Lau, C.C.Y., Lu, L., Liu, Q., Wang, L., Feng, Y., Wang, Y., et al. (2014). Exceptionally potent neutralization of Middle East respiratory syndrome coronavirus by human monoclonal antibodies. J Virol 88, 7796-7805.

Zaki, A.M., van Boheemen, S., Bestebroer, T.M., Osterhaus, A.D.M.E., and Fouchier, R.A.M. (2012). Isolation of a novel coronavirus from a man with pneumonia in Saudi Arabia. N Engl J Med 367, 1814-1820.

Zhao, G., Du, L., Ma, C., Li, Y., Li, L., Poon, V.K., Wang, L., Yu, F., Zheng, B.J., Jiang, S., et al. (2013). A safe and convenient pseudovirusbased inhibition assay to detect neutralizing antibodies and screen for viral entry inhibitors against the novel human coronavirus MERS-CoV. Virol J 10, 266.

\section{SUPPORTING INFORMATION}

Figure S1 The functional verification of MERS-GD27 in vitro.

The supporting information is available online at http://life.scichina.com and https://link.springer.com. The supporting materials are published as submitted, without typesetting or editing. The responsibility for scientific accuracy and content remains entirely with the authors. 